\title{
Upscaling of dilution and mixing using a trajectory based Spatial Markov random walk model in a periodic flow domain
}

\author{
Nicole L Sund ${ }^{\mathrm{a}, \mathrm{c}}$, Giovanni M Porta ${ }^{\mathrm{b}}$, Diogo Bolster ${ }^{\mathrm{c}}$ \\ ${ }^{a}$ Division of Hydrologic Sciences, Desert Research Institute, Reno, NV 89512, USA \\ ${ }^{b}$ Dipartimento Ingegneria Civile ed Ambientale Politecnico di Milano, Piazza L. Da Vinci, \\ 32, I-20133 Milano, Italy \\ ${ }^{c}$ Dept. of Civil and Environmental Engineering and Earth Sciences, University of Notre \\ Dame, IN, USA \\ ddbolster@nd.edu
}

\begin{abstract}
The Spatial Markov Model (SMM) is an upscaled model that has been used successfully to predict effective mean transport across a broad range of hydrologic settings. Here we propose a novel variant of the SMM, applicable to spatially periodic systems. This SMM is built using particle trajectories, rather than travel times. By applying the proposed SMM to a simple benchmark problem we demonstrate that it can predict mean effective transport, when compared to data from fully resolved direct numerical simulations. Next we propose a methodology for using this SMM framework to predict measures of mixing and dilution, that do not just depend on mean concentrations, but are strongly impacted by pore-scale concentration fluctuations. We use information from trajectories of particles to downscale and reconstruct pore-scale approximate concentration fields from which mixing and dilution measures are then calculated. The comparison between measurements from fully resolved simulations and predictions with the SMM agree very favorably.
\end{abstract}

Keywords: Dilution and Mixing, Upscaling, Spatial Markov Model

\section{Introduction}

Predicting transport of chemical species in flows through porous media is challenging due to complex non-uniform flows at pore scales [48]. While ad-

Preprint submitted to Advances in Water Resources

February 27, 2017

(C) 2016. This manuscript version is made available under the Elsevier user license http://www.elsevier.com/open-access/userlicense/1.0/ 
vances have been made in effective models that predict downstream transport of conservative species, open challenges remain to predict the transport of reactive species at large scales [27]. In particular, when predicting transport of reactive species it is typically not sufficient to develop a model that can predict mean concentrations, but one that can also incorporate subscale fluctuations $[27,6]$. Reactions can be highly localized events, governed by complex nonlinear dynamics, meaning that fluctuations about the mean do not trivially average out when upscaling. Indeed accurately capturing fluctuations of concentrations about mean values is key to predicting mixing driven reactions in setups ranging from simple closed systems [5, 46, 10], non-uniform flows [45], one dimensional porous columns, [31, 30, 28, 24] and highly heterogeneous porous media [42]. Any sound effective model should be able to accurately predict mixing processes, at the very least in some global sense, to have a hope of predicting the amounts of mixing driven reaction that occur.

Effective models that predict mean concentrations in complex flows date back to the seminal work of GI Taylor [63]. He showed that after sufficient time, three dimensional transport in laminar flow through a tube can be described by a one dimensional advection dispersion equation with an effective dispersion coefficient. These ideas were generalized to more complex flows via the method of moments [14], volume averaging [47] and homogenization [32], among others. This model also grounds the concept that solute dispersion in porous media can be treated via a Fickian model, i.e. with a constant dispersivity parameter [3].

Taylor dispersion models have proved indispensable and are used to great effect across the hydrologic sciences and beyond [e.g. 20, 21, 4, 33, 23, 13, 58, 9, 53]. As with all models, they are built on assumptions and have limits. Strictly, these ideas are only valid at large times, typically defined as times greater than $\tau_{D}=L^{2} / D$, where $L$ is a characteristic length scale and $D$ the diffusion coefficient [57]. This is the characteristic time for molecular diffusion to homogenize concentration over length scale $L$. From the perspective of predicting mixing this is also useful since at these late times mean concentration is representative of actual concentrations; that is, fluctuations about the mean are small. 
Thus at these late times, knowing the mean (spatially averaged) concentration is sufficient to quantify most features of transport. Depending on the system in question, this characteristic time can be prohibitively long and difficult to estimate. Additionally there is abundant evidence that in real complex porous media advection velocities span orders of magnitude and that structural complexity makes it impossible to define a unique characteristic length scale [e.g. $7,59]$. Thus the existence of a unique Péclet number to delineate advection and diffusion dominance is questionable [50]. This also implies that characteristic time $\tau_{D}$ at which a transition to a Fickian transport regime can be expected is unknown a priori and may be quite long.

At times before this characteristic time, significant subscale fluctuations in concentration can exist, which impact the prediction of longitudinal downstream transport, mixing and reaction processes [e.g. 43, 11, 52, 51]. Several efforts have extended the above mentioned approaches to predict mean concentrations at pre-asymptotic times, [44, 64, 55] as well as mixing processes [11]. While powerful, other studies have shown that many assumptions required to close these models are restrictive, confining regimes of validity $[2,1]$.

An alternative upscaling approach to those mentioned above, built from a Lagrangian perspective, is the Spatial Markov model (SMM), first proposed by Le Borgne et al. [40] to model effective transport in highly heterogeneous porous media. The SMM is a random walk model that describes a plume in terms of a large number of discrete particles that transition through space and time following a set of specific probabilistic rules; the SMM, unlike other random walk models, fixes the size of spatial jumps particles make and then enforces correlations between successive transitions, a step that is essential to accurately capturing effective behaviour, particularly for advection dominated systems [10]. As with most upscaling efforts one of the goals with the SMM is a model that can be run efficiently at larger scales than the representative elementary volume and computational savings with the SMM can often be on the order of several orders of magnitude in terms of computational time [60]. The SMM is also built on assumptions that restrict its regime of validity; e.g. it can only be applied 
at spatial scales where correlation effects are monotonically decreasing in an exponential manner [61]. However, it can typically be applied at temporal and spatial scales much smaller than those set by the characteristic Taylor dispersion time $\tau_{D}$ [61]. The SMM has to date been successfully applied to predict mean transport of conservative solutes across a broad range of flows, ranging in complexity and scale $[40,41,35,43,25,10,60,36,34]$. While it has been extended to include heterogeneous first order reactions [62], to date mixing, and by extension mixing driven reactions, have not been explicitly included.

Here we propose a novel methodology with a twofold aim: (i) model longitudinal transport in periodic domains and (ii) extend the SMM to model dilution and mixing. We apply our approach to a model problem characterized by a simple spatially periodic domain, described in Section 2. Note that many approaches, including the aforementioned method of moments and volume averaging, typically involve solving a closure problem assuming some periodic representative elementary volume. As discussed extensively in the literature, this should not be regarded as a restriction of the approach [e.g. 54, 65]. To date the SMM for a periodic system is parameterized by measuring the travel time distribution of particles across two successive periodic elements and measuring correlation effects via a transition matrix [40]. We argue that, specifically for the case of a spatially periodic domain, this effort can be reduced to measuring particle trajectories across a single periodic element. Then, we couple our longitudinal transport model with an additional procedure to predict dilution and mixing of solute mass in the periodic domain. Both the longitudinal transport and mixing models are obtained by exploiting information on pore-scale particle trajectories, as detailed in Section 3.

\section{Model System}

In this paper we focus on a particular unit cell geometry, a channel with sinusoidal boundaries, which in the context of porous media was first studied by [29] and [15]. While simple, it has been shown to capture some very rich 


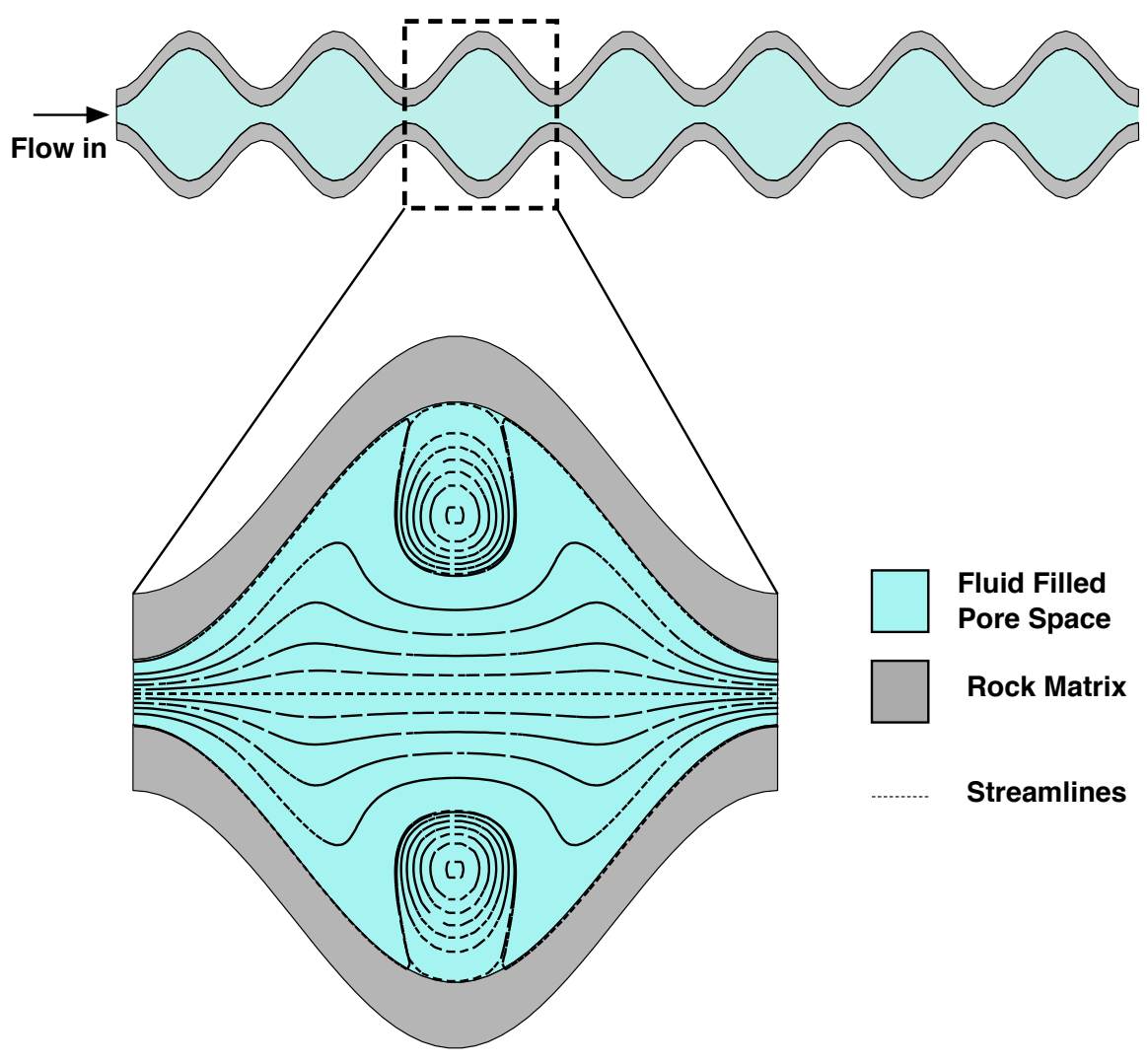

Figure 1: Schematic of flow domain (top) and the unit cell 'pore' (bottom) for flow and transport modeling in this work. Streamlines for the flow are calculated using the semianalytical solution for Stokes flow developed by [38]. 
dynamics, representative of complexities in real porous media. Multiple studies have used this, or very similar, geometries to explore moderate to high Reynolds number flow and transport $[18,19,12,10,55]$ and to study the impact of geometry on macroscopic dispersion $[17,8,16,43]$ and dilution [15]. The system is well studied and serves as an ideal benchmark against which to test our modeling approach.

\subsection{Geometry \& Flow}

The flow domain used in this study is shown in figure 1. The walls of the channel are described by $h(x)=\bar{h}+h^{\prime} \sin \left(\frac{2 \pi x}{L}-\frac{\pi}{2}\right)$, where $x$ is the horizontal coordinate, $h(x)$ is the half-aperture, $\bar{h}$ is the mean half-aperture, $h^{\prime}$ is the amplitude fluctuation of the half-aperture and $L$ is the length of a single cell. Here, we focus on the case where $\bar{h}=L / 4$ and $h^{\prime}=0.8$. Figure 1 also depicts the streamlines for the flow, calculated using a semi-analytical solution [38, 29]. The solution assumes Stokes flow, with Reynolds number much less than one, meaning inertial effects are negligible, which is generally a good approximation for porous media. Details on the flow solution are available from multiple sources $[38,29,8]$ and so are not presented here.

\subsection{Simulation of Transport at the Microscale}

We consider transport governed by the advection diffusion equation at the pore-scale,

$$
\frac{\partial \widehat{C}(\widehat{\mathbf{x}}, t)}{\partial t}+\widehat{\mathbf{u}}(\widehat{\mathbf{x}}) \cdot \nabla \widehat{C}(\widehat{\mathbf{x}}, t)=D \nabla^{2} \widehat{C}(\widehat{\mathbf{x}}, t)
$$

where $\widehat{C}(\widehat{\mathbf{x}}, t)$ is concentration, $\widehat{\mathbf{u}}(\widehat{\mathbf{x}})$ is the advective velocity field, and $D$ is the diffusion coefficient. Hats over the symbols mean that we are referring to quantities defined at the pore scale. Boundary conditions are no-flux at the walls. In all cases we consider a pulse initial condition, flux weighted along the pore throat. Note also, that due to the natural symmetry of the system about $y=0$ we will only ever simulate half of the domain, imposing a symmetry (no flux) condition at $y=0$. 
To solve this system, we implement a random walk [56], where the plume is discretized into $N$ particles. For a time step $\Delta t$, particle $i$ moves as

$$
\begin{aligned}
& \widehat{x}_{i}(t+\Delta t)=\widehat{x}_{i}(t)+\widehat{u}_{i} \Delta t+\xi_{i} \sqrt{2 D \Delta t} \\
& \widehat{y}_{i}(t+\Delta t)=\widehat{y}_{i}(t)+\widehat{v}_{i} \Delta t+\eta_{i} \sqrt{2 D \Delta t}
\end{aligned} \quad i=1, \ldots, N,
$$

where $\widehat{x}_{i}$ and $\widehat{y}_{i}$ are the horizontal and vertical position of particle $i$ respectively and $\xi$ and $\eta$ are independent identically distributed Gaussian variables with zero mean and unit variance. No flux boundaries are modeled by elastic reflection.

We use a Lagrangian random walk because for periodic flow domains it is possible to consider transport over large distances through many unit cells without the need for prohibitively large numerical meshes, as only a particle's position relative to the unit cell is ever needed. Additionally, for sufficiently smooth velocity fields, random walk methods do not suffer numerical dispersion. For all cases one million particles were used with time steps of $\Delta t=10^{-3}$, which for this geometry have been shown to produce converged results [8].

Concentrations are calculated by discretizing the space into square cells with sides of length $\Delta x$, counting the number of particles within each cell $\left(n_{p}\right)$, and calculating $\widehat{C}(\widehat{\mathbf{x}})=\frac{n_{p} m_{p}}{V}$, where $V=\Delta x^{2}$. For boundary cells which intersect the wall it is straightforward to adjust the volume of fluid accordingly. The mass of a particle $m_{p}=1 / N$ as we inject a pulse of unit mass in all instances considered here. In general, we used a value of $\Delta x=0.02$ for the spatial grid. This value was chosen based on a convergence test whereby results were invariant when the grid spacing was halved or particle numbers doubled. The same grid and formula described above is used to calculate concentration as well as dilution and mixing metrics for our fully resolved simulations, denoted by variables with hats, as well as our effective modified SMM, which will be denoted by variables with tildes and are described in detail below in section 3 .

This system is primarily characterized by the dimensionless Péclet number, $P e=\frac{2 \bar{h} \bar{u}}{D}$. While the flow solution assumes small Reynolds number $R e<1$, the Péclet number for many solutes in water is typically 2-3 orders of magnitude 
larger, meaning that while flow may be viscosity dominated, transport can be advection dominated with typical values of $0.1<P e<10^{3}$ [29]. Here we focus on $P e \geq 100$, as these cases can be harder to upscale since the asymptotic time and length scales where Taylor dispersion works are largest. In all cases $P e$ is tuned by setting $\bar{u}=1,2 \bar{h}=1$ (in arbitrary units) and choosing $D$ appropriately.

\subsection{Observables to Test the Effective Model}

Here we define several observables that we will measure explicitly from high resolution direct numerical simulations, using the random walk method in (2). These observables will be treated as the ground truth against which our effective model will be tested for validation.

\subsubsection{Breakthrough Curves}

To test our model's ability to capture mean effective transport, the observable we will focus on is a concentration breakthrough curve (BTC). BTCs at multiple downstream distances of $x=5 L, 10 L, 25 L$ and $50 L$ will be measured. Several locations are chosen so as to test the veracity of the model. An ability to capture one breakthrough curve can be achieved by parameter tuning, but being able to accurately capture BTCs at all four locations suggests a robustness that only a physically consistent model should be able to capture [26]. For example, a model may be able to interpret the transport behavior at the smallest distance or times, but might fail to make accurate predictions at larger space-time scales or vice versa [e.g. 40, 25].

\subsubsection{Dilution Index and Scalar Dissipation}

As outlined in the introduction one of the objectives of this work is to extend the Spatial Markov model in such a way as to predict mixing. To this end, we measure the temporal evolution of the dilution index [37] as well as the second concentration moment, the time derivative of which is related to another known

metric for mixing, the scalar dissipation rate [39, 11, 49]. For the considered pulse initial condition, the dilution index is defined by, 


$$
E(t)=\exp \left(-\int C(\mathbf{x}, t) \log [C(\mathbf{x}, t)] d \mathbf{x}\right)
$$

and the second concentration moment as,

$$
M(t)=\int C^{2}(\mathbf{x}, t) d \mathbf{x}
$$

where $C(\mathbf{x}, t)$ refers generically to either the microscale concentration, $\widehat{C}(\widehat{\mathbf{x}}, t)$ or the SMM predicted concentration, $\tilde{C}(\mathbf{x}, t)$ that will be defined in section 3 . The scalar dissipation $\xi$ rate is closely related to this measure as $\xi=-\frac{d M(t)}{d t}$.

\section{Transport and Mixing - A Spatial Markov Model}

A common goal of effective transport models, such as those discussed in the introduction, is to reduce the dimensionality of the transport problem to a single dimension in the direction of flow. To that end, here we propose and describe an SMM. As with any random walk, we discretize our solute into a large number of particles and then have them march through space and time following a defined set of deterministic and stochastic rules. For our Spatial Markov model we fix the size of the spatial jump that a particle makes and treat the amount of time the particle takes to make that jump as random. At step $k$, a particle's state is defined by its position $\tilde{x}_{i}^{k}$ and time $\tilde{t}_{i}^{k}$. Note that, as desired, vertical location is projected out and thus this framework without further modification can only provide a representation of vertically averaged concentration. The equation for transport, a Langevin equation that updates space and time of each particle, is given by

$$
\begin{aligned}
\tilde{x}_{i}^{k+1} & =\tilde{x}_{i}^{k}+L \\
\tilde{t}_{i}^{k+1} & =\tilde{t}_{i}^{k}+\tau_{i}^{k} .
\end{aligned}
$$

Consistent with previous works $[62,43,60,10]$, for our SMM, $L$ is a spatial 
jump of fixed size that particles make during any step; for a periodic domain, the length of the periodic unit cell, or integer multiple thereof, seems most appropriate. The random component in (5) is the time $\tau$ required for each particle to travel the distance $L$. This reflects the broad distribution of transport velocities, typical within a porous domain. The distribution from which this time step is sampled, is given by the travel time distribution that it takes particles to traverse one unit cell of length $L$.

Some of the most common implementations of (5) are random walks where successive $\tau$ 's are independent and identically distributed, but increasingly models where successive steps are correlated are becoming commonplace. Here we advocate for the case where successive steps are correlated, which has been shown to be particularly important in highly advection dominated systems $(P e>O(100))[10,62,61]$. To date correlations have been most typically imposed using the idea of a transition matrix [40]. Here, we will take a slightly different approach, taking advantage of the spatially periodic nature of our domain. Moreover, our method provides a novel approach to predict the evolution of mixing in time. To summarize, the key novel elements of our procedure, are (i) the methodology employed to compute the travel times $\tau_{i}{ }^{k}$ and (ii) that we provide a downscaled approximation of the position of the particle $(\tilde{x}(t), \tilde{y}(t))$ from which to estimate mixing (e.g., through (3)-(4)) not included in currently available SMM implementations. The following sections present the parameterization and the implementation of our proposed approach.

\subsection{Characterization of pore-scale transport trajectories}

Before describing our novel approach, let us first explain why correlation between successive steps is important. To this end, consider the sample particle trajectories that are depicted in figure 2 and which are obtained through simulation of pore-scale transport in the model geometry using the random walk technique described in section 2.2. The blue and red lines depict the trajectories of two particles injected into the fastest moving part of the flow along the center line of the channel. This suggests both particles traverse the first unit 


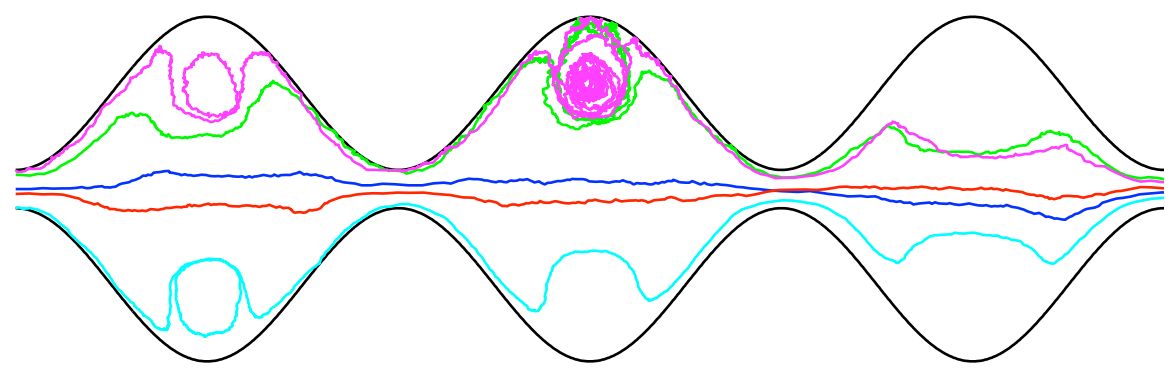

Figure 2: Sample particle trajectories for $P e=1000$

cell very quickly and then, due to the fact that they continue on comparably fast streamlines, they pass through the second and third unit cell quickly also. The mechanism that will cause these particles to eventually jump to a slow trajectory is diffusion across streamlines. However, the local impact of diffusion is considerably smaller with respect to advection, given that we consider here a largely advection-dominated case $(P e=1000)$. Likewise, the slowest trajectories represented in figure 2 (i.e. purple, cyan and green) persist in being slow across successive pores.

The parameterization of the SMM is typically performed using a statistical characterization of particle travel times, obtained by simulating pore-scale transport of a set of particles across a representative unit cell. In previous studies correlations have been imposed by measuring the travel time statistics of particles across two successive unit cells and building a transition matrix to represent jumps between travel time classes [40]. While this method has been shown to provide a robust characterization of the distribution of the travel times, it requires simulation across two successive elements. Here we propose an alternative method that only requires simulation across a single element. The key to our approach is to recognize that what sets a particle's travel time across a periodic element is its vertical location at the inlet. Based on where it starts its journey across the pore, a particle has a finite range of travel times 
as well as possible vertical locations where it will exit the pore. By virtue of the fact that these are non-inertial memoryless particles, the vertical location where it exits the previous pore serves as the vertical location from where it starts its next transition. Figure 3 highlights the idea by showing three sets of particle trajectories originating from three inlet locations, which are identified by different colours. Results for $P e=100$ and $P e=1000$ are shown. For the smaller Péclet number, while all trajectories identified by the same colour start at the same inlet location, at the outlet they can span a broad range of outlet locations. For the larger Péclet number the outlet locations associated with a single inlet location are much less disperse, due to less relative influence of diffusion. Almost no overlap between the three sets of trajectories are observed when $P e=1000$.

As anticipated above, the parameterization of the SMM requires the simulation of pore-scale transport for a number of particles $N_{P S}$. This implicitly defines a set of $\mathcal{S}=\left\{s_{1}, \ldots, s_{N_{P S}}\right\}$ of trajectories $s_{i}$, which all travel from the inlet to the outlet cross sections of the unit cell. Our model entirely relies on the information carried by $s_{i}$. Each trajectory $s_{i}$ is directly associated with an inlet location $y_{i n}\left(s_{i}\right)$, an outlet location $y_{\text {out }}\left(s_{i}\right)$ and a travel time $\tau\left(s_{i}\right)$. Moreover, we define a specific order of the trajectories $s_{i}$ by setting $y_{\text {in }}\left(s_{1}\right)<y_{i n}\left(s_{2}\right)<\ldots<y_{i n}\left(s_{N_{P S}}\right)$. Relying on this ordering, the trajectories are subdivided into a number of $N_{b i n}$ subsets,

$$
\mathcal{S}\left(j_{b i n}\right)=\left\{s_{i} \in \mathcal{S}: y_{i n}\left(s_{j b-}\right) \leq y_{\text {in }}\left(s_{i}\right) \leq y_{i n}\left(s_{j b+}\right)\right\}
$$

where

$$
j_{b-}=\left(\frac{j_{b i n}-1}{N_{b i n}}\right) N_{P S}+1 ; \quad j_{b+}=\frac{j_{b i n}}{N_{b i n}} N_{P S} ; \quad j_{b i n}=1, \ldots, N_{b i n}
$$

Definition (6) introduces a mapping between any location $y_{\text {in }}$ at the inlet section and a bin number $j_{b i n}$ which will be key to parameterize transport in our SMM. Note that, while other definitions are possible, according to (7) the 
number of trajectories is the same for each subset $\mathcal{S}\left(j_{b i n}\right)$. In the numerical examples presented below we apply these definitions, fixing $N_{P S}=10^{6}$ and $N_{\text {bin }}=10^{2}$, so that each subset $\mathcal{S}\left(j_{\text {bin }}\right)$ comprises $10^{4}$ trajectories.

The definitions provided establish a direct link between the location of the particle at the inlet section and the travel time through the trajectory $s_{i}$. As discussed above, the outlet position associated with trajectory $s_{i}$ serves as the inlet position for the next transition. The rightmost column of Figure 3 demonstrates the correlation between successive inlet positions, by showing the probability of jumping from one bin to another in two successive cells. This representation resembles the transition matrices which are typically employed in previous implementation of the SMM for longitudinal transport. It should be noted, however, that we will never actually use this matrix in our model and its representation in Figure 3 is merely intended to demonstrate the influence of correlation. For $P e=100$ the transition probabilities are almost uniform, while there is a strong signal of correlation associated with $P e=1000$; particles which start close to the center of the considered wavy channel are most likely to have their next transition close to the center too, while particles closer to the boundary are likelier to have their next transition start there. This merely quantifies the already discussed qualitative effect shown in the middle column of the figure. It is worth noting that these transition matrices need not be symmetric, as they clearly are not here. The threshold of $P e>O(100)$ for correlation to become important is very much in line with previous studies [10, 62, 61].

Our idea is to further employ the information contained in trajectories $s_{i}$ to predict the evolution of mixing within the system. This, due to the nonlinear dependence on concentration evident from equations (3) and (4), requires knowledge of subscale fluctuations in concentration; merely knowing average concentrations does not suffice. Thus, one possibility is to downscale information from our upscaled model back down to a more resolved smaller scale at locations in space and time where we need it. A distinctive feature of our proposed Spatial Markov model with respect to previous implementations is that we focus on sampling particle trajectories. It is logical to continue within this 

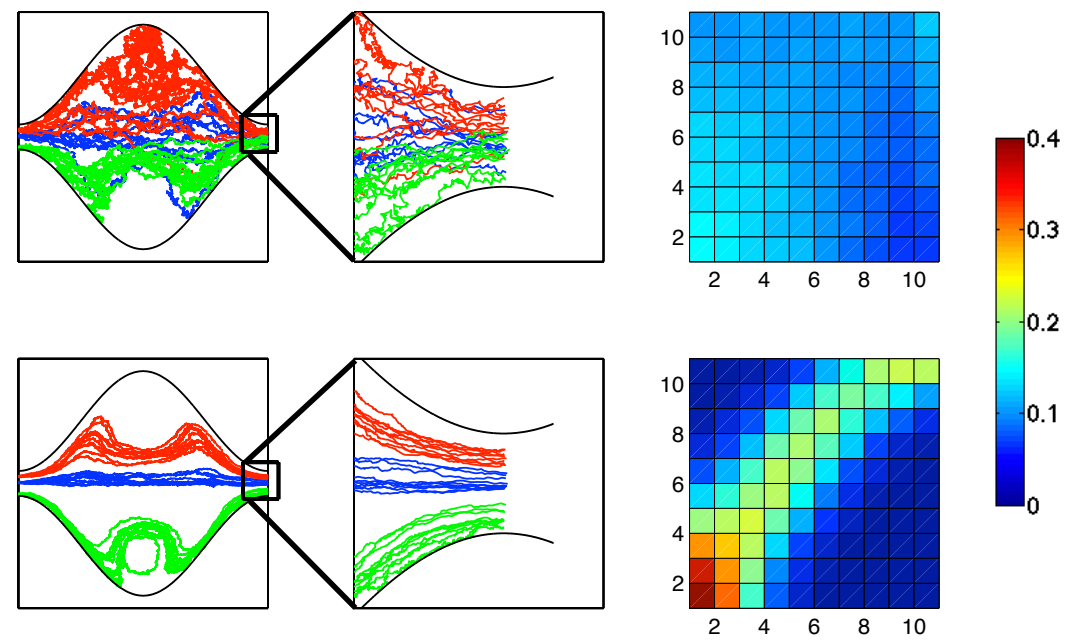

Figure 3: (left) Sample particle trajectories for $P e=100$ (top) and $P e=1000$ (bottom) with a zoomed in region where particles exit the domain. Blue trajectories start in the fastest region of the pore throat, red an intermediate region and green the slowest region. (right) The corresponding transition matrices with 10 bins depicting the probability of transitioning to a new bin of inlet locations. 
framework and encode in our model more information on particle trajectories $s_{i}$, rather than just focusing on travel times alone. To this end, we store a discretized counterpart of the trajectory $s_{i}$ which allows us to reconstruct the path of $s_{i}$ inside the cell and the related travel time. For each trajectory we introduce a discretized counterpart which can be defined in matrix format,

$$
\mathbf{P}\left(s_{i}\right)=\left[\begin{array}{llll}
\chi_{1} & \chi_{2} & \ldots & \chi_{n} \\
\eta_{1} & \eta_{2} & \ldots & \eta_{n}
\end{array}\right],
$$

where the pairs $(\chi, \eta)_{\omega}$ identify the locations along the trajectory $s_{i}$ which correspond to a travel time equal to $\frac{\omega}{n} \tau\left(s_{i}\right)$ with $\omega=1, \ldots, n$. Here, $n$ is the chosen number of intervals with which to approximate each trajectory and is a key parameter of our discretization approach. Note that our methodology yields positions along the trajectory which are equidistributed in time. In order to downscale the positions along the trajectories at intermediate times, we linearly interpolate between the positions stored in $\mathbf{P}\left(s_{i}\right)$ in order to obtain the complete mapping between the travel time domain and the location along the trajectory.

Figure 4 displays what some sample interpolated trajectories look like relative to actual trajectories for a variety of possible paths. For the intents of this paper we found that reasonable results can be obtained with $n=20$ (see figure 4 ), although this can cause small problems in regions where the velocities are largest in the pore throats (see for example that some interpolated trajectories actually leave the domain) and so more may be needed. As can be seen, for the larger value of $n$, the paths are reconstructed almost perfectly, while for the lower value of $n$ some clear issues arise (e.g., some of the trajectories cross the liquid-solid boundary). In light of this observation, we identify the number of discretization points $n$ as a key numerical parameter for our methodology.

\subsection{Modeling procedure}

In this section we describe how the information on pore-scale trajectories described in section 3.1 is practically employed to simulate the evolution of 


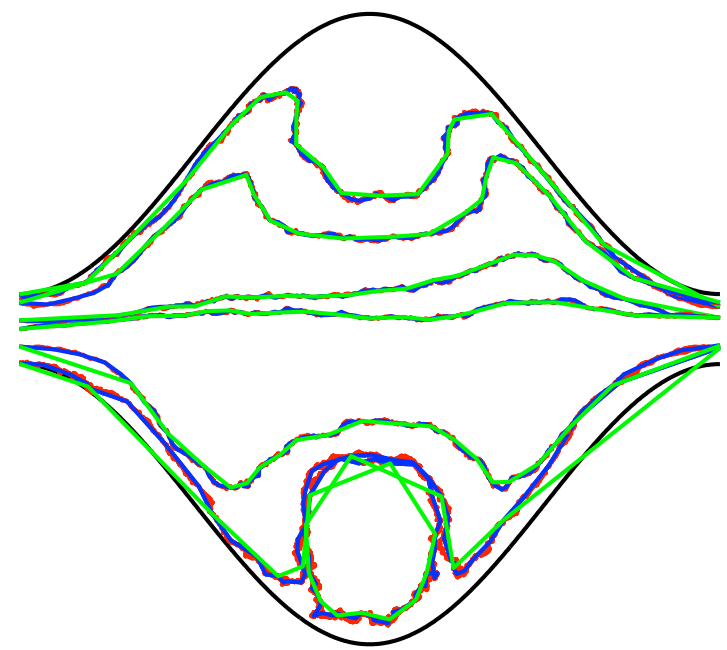

Figure 4: Sample particle trajectories $s_{i}$ - with subsampled interpolations $P\left(s_{i}\right)$ superimposed. The red lines are the actual particle trajectories, while the green and blue lines are the interpolated paths with $n=20$ and $n=50$ respectively.

solute concentration and of mixing. The numerical procedure is represented schematically in a flow chart in Figure 5.

Our SMM considers transport of a set of $N_{p}$ particles within a time window $t \in\left[0, T_{\text {end }}\right]$. In the following, we present separately the modeling steps associated with longitudinal transport of particles in the SMM, represented in blue in Figure 5, and those related to the downscaling procedure employed to approximate mixing, represented in green in Figure 5 .

\subsubsection{Longitudinal transport}

To exemplify our proposed modeling strategy, let us consider transport of a particle $p_{i}$ which is introduced in the system at the inlet boundary, located at $x=0$. In the SMM we assign to $p_{i}$ an initial location $y_{i n}^{0}$. Here the location $y_{i n}^{0}$ is selected to obtain a flux-weighted injection, consistent with the implemented direct numerical simulation (DNS). This location can then be used to assign the particle to a bin $j_{b i n}$ through (6), using the trajectories $s_{i}$ obtained from the pore-scale characterization of transport in the unit cell. Then, we randomly select a trajectory $s^{1}\left(p_{i}\right)$ from the set $\mathcal{S}\left(j_{\text {bin }}\right)$ and set that the transition $k=1$ 
of particle $p_{i}$ happens along $s^{1}\left(p_{i}\right)$. The travel time associated with the first transition of particle $p_{i}$ is consequently assigned as $\tau^{1}\left(p_{i}\right)=\tau\left[s^{1}\left(p_{i}\right)\right]$ in (5). At the next time step the same procedure can be followed upon assigning $y_{i n}^{1}=$ $y_{\text {out }}\left[s^{1}\left(p_{i}\right)\right]$ and so on for all steps $k$, until the desired number of transitions has occurred. This procedure is graphically sketched by the blue loop in Figure 5 .

\subsubsection{Dilution and mixing}

We define a user-defined set of times $t_{M}=\left\{t_{M, 1}, \ldots, t_{M, N_{M}}\right\}$, at which we aim to evaluate metrics (3) and (4). We illustrate here the procedure employed to retrieve the approximate position of particle $p_{i}$ in the system at a generic time $t_{M, j}$, which is labeled as $\left[\tilde{x}_{i}, \tilde{y}_{i}\right]_{j}$. First we march the particle through $k+1$ steps such that $\tilde{t}^{k}<t_{M, j} \leq \tilde{t}^{k+1}$. Then we compute which locations along the discretized trajectory the particle is between based on the fraction of the total travel time which is associated with the time difference $t_{M, j}-\tilde{t}^{k}$, i.e.,

$$
\omega=\operatorname{floor}\left(n \frac{t_{M, j}-\tilde{t}^{k}}{\tilde{t}^{k+1}-\tilde{t}^{k}}\right)
$$

where floor rounds down to the lower integer. We then employ the matrix $\mathbf{P}\left(s^{k}\right)$ (equation (8)) to obtain the position of the particle within the cell. According to the discretization of the particle pathways introduced in section 3.1 (see Figure 3), the particle lies somewhere between $(\chi, \eta)_{\omega}$ and $(\chi, \eta)_{\omega+1}$. We then define,

$$
\epsilon=\frac{t_{M, j}-\tau \omega / n}{\tau / n},
$$

to linearly interpolate the position of $p_{i}$ such that,

$$
\left[\tilde{x}_{i}, \tilde{y}_{i}\right]_{j}=(k L, 0)+(\chi, \eta)_{\omega}+\epsilon\left((\chi, \eta)_{\omega+1}-(\chi, \eta)_{\omega}\right) .
$$

The particle position is then stored and can be employed to reconstruct an

approximated concentration field $\widetilde{C}\left(x, y, t_{M, j}\right)$. Dilution and mixing can be 
then quantified upon substituting $\widetilde{C}\left(x, y, t_{M, j}\right)$ in (3) and (4). The procedure is represented in the green loop in Figure 5.

Note that the transport model identified by the blue loop is stand-alone, i.e., it can be run independently from the evaluation of mixing and dilution. On the other hand, the procedure employed to predict mixing (green loop) is fully nested within the transport modeling procedure (blue loop), as it relies on the information associated with the trajectories, which are selected along the transport simulation.

\section{Results}

\subsection{Breakthrough Curves}

Figure 6 depicts breakthrough curves located at distances $5 L, 10 L, 25 L$ and $50 \mathrm{~L}$ downstream of the injection location. Two sets of data are shown corresponding to the measurements from the small scale direct numerical solutions as well as predictions with our novel SMM. The agreement between the two can be seen to be very good with the SMM predictions matching well at all times and all locations. This is the case for both Péclet numbers. Our modified SMM, designed for periodic flow domains, appears to match mean effective behavior well, working comparably to previous SMM models applied to similar geometries [e.g. 43, 10]. It is also worth noting that the computational time for running the SMM to produce these curves is on the order of 100 times faster than the DNS results, although neither code was fully optimized for performance. Note that it is also straightforward to use this framework to calculate effective longitudinal dispersion coefficients, which compare favorably with predictions using classical theories and measurements from DNS simulations [e.g. 43, 10, 60, 62].

\subsection{Scalar Dissipation and Dilution Index}

Figure 7 depicts the evolution over time of the dilution index as well as the second concentration moment, the two observables we identified for mixing and dilution. In the same way as for the BTCs in the previous subsection, two sets 


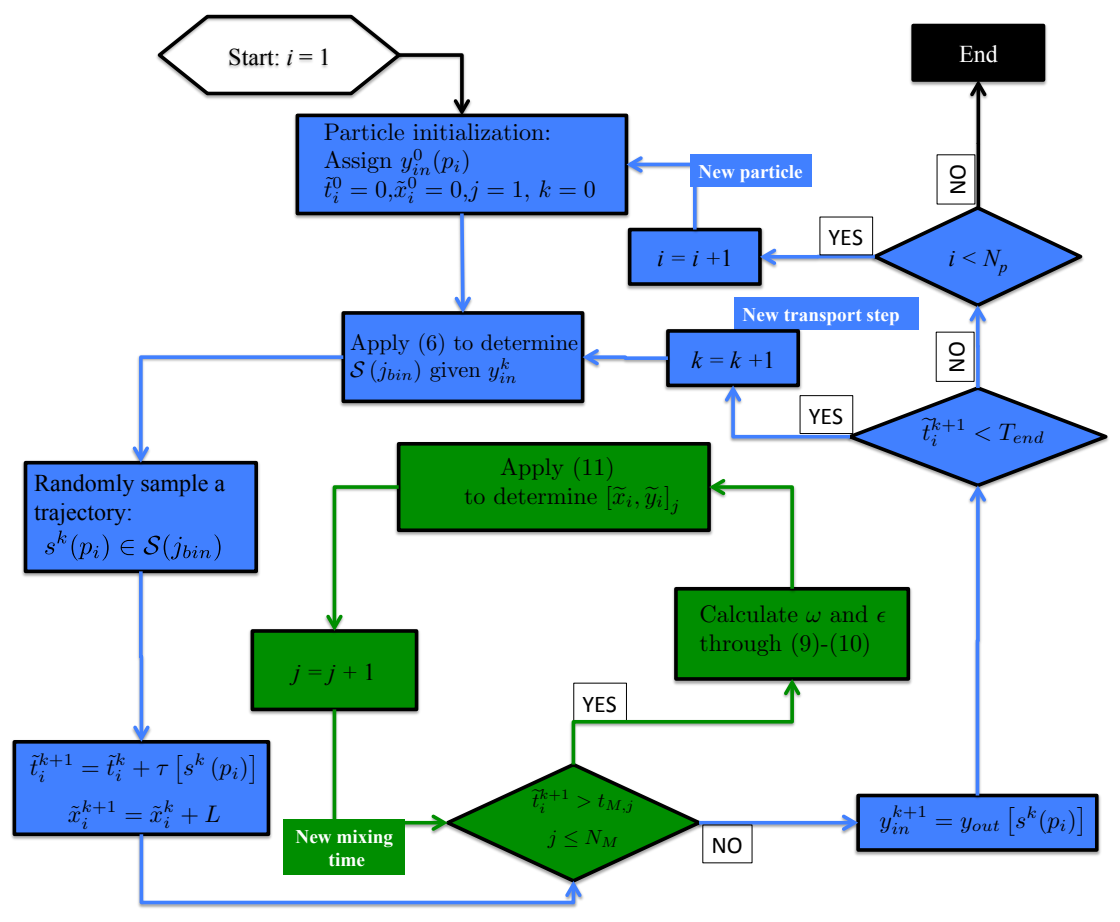

Figure 5: Algorithm to predict one-dimensional longitudinal transport (blue loop) and mixing evolution (green loop). $i, j$, and $k$ are indices of particles, time steps, and longitudinal transport steps, respectively. Thus $\tilde{x}_{i}^{k}$ and $\tilde{t}_{i}^{k}$ are the longitudinal spatial location and the temporal location of the $i$ th particle at the $k$ th longitudinal transport step. Based on which bin the particle is in, $j_{b i n}$, at each modeling step particle $i\left(p_{i}\right)$ chooses a trajectory $\left(s^{k}\left(p_{i}\right)\right)$ to follow from the set of trajectories in its bin $\left(\mathcal{S}\left(j_{b i n}\right)\right)$. This trajectory sets the particle's travel time and ending position $\left(y_{i n}, y_{o u t}, \tau\right)$. The time level $t_{M, j}$ is from a set of user defined times to measure the evolution of mixing, the times at which we find the particle's estimated position along its trajectory through interpolation. 

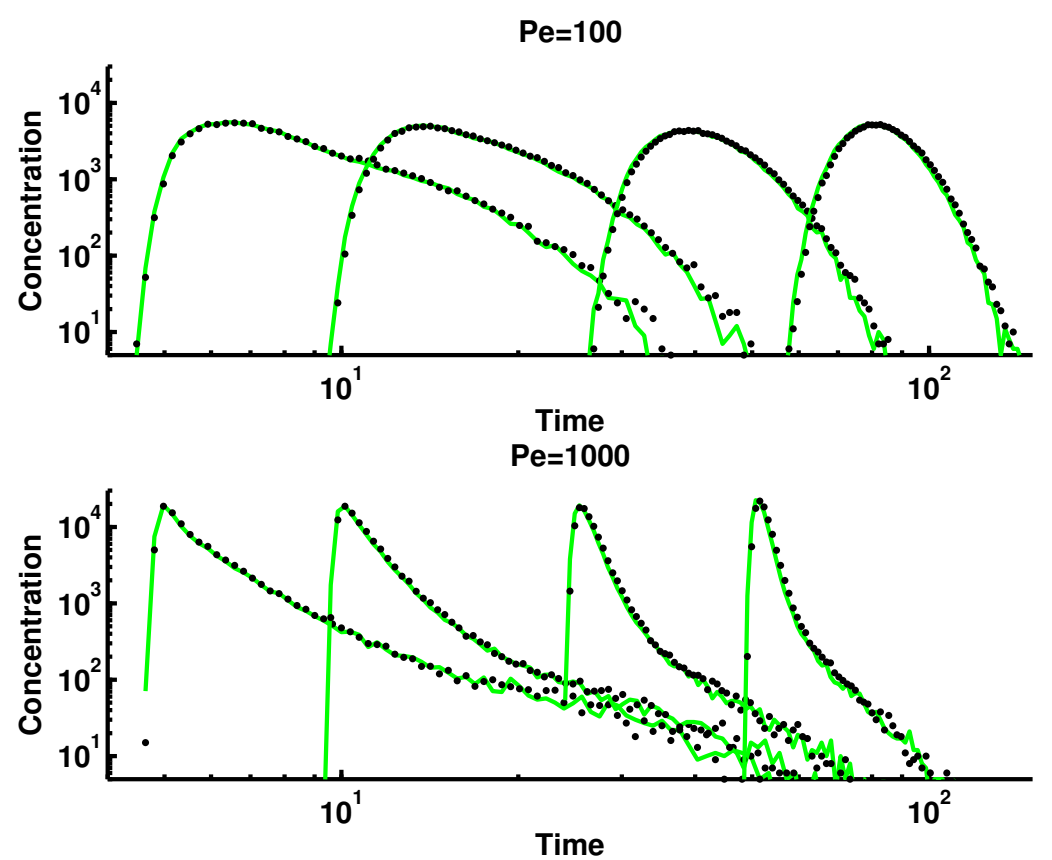

Figure 6: Breakthrough Curves for two Péclet numbers, $P e=100$ (top) and 1000 (bottom), comparing measurements from the high resolution direct numerical simulations (green solid line) to predictions by the SMM model (black dots). Breakthrough curves are at distances $x=5 L, 10 L, 25 L$ and $50 L$. 

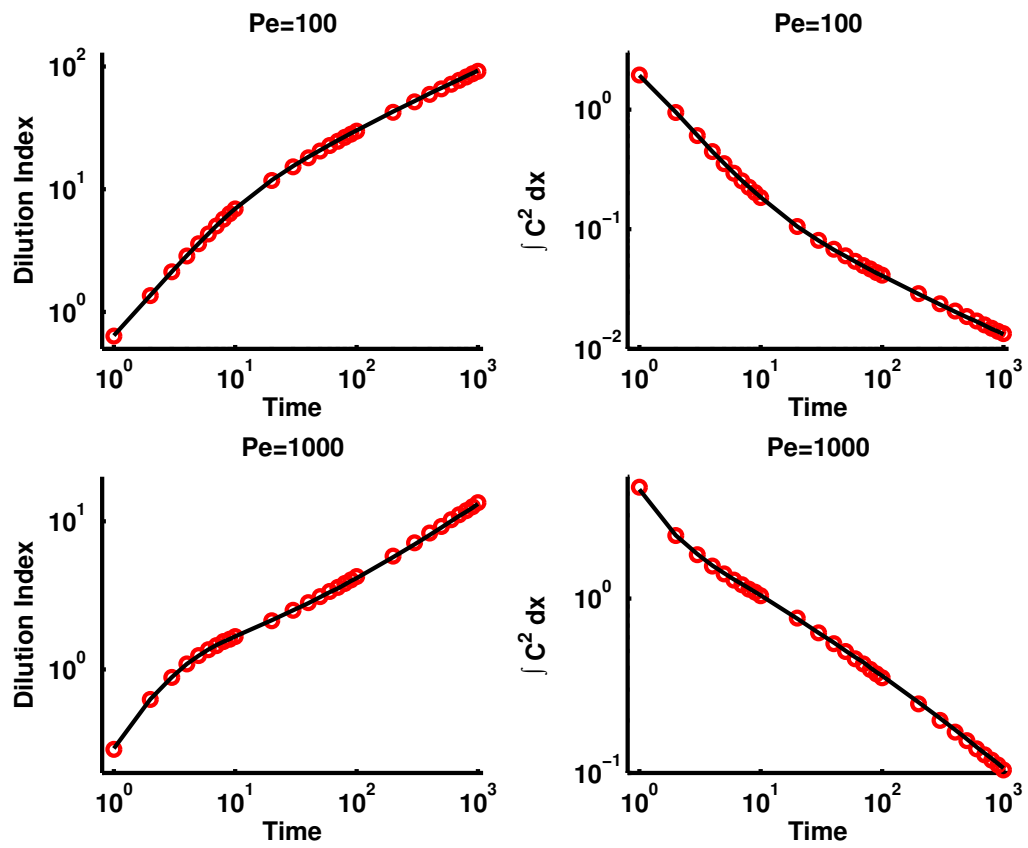

Figure 7: A comparison of the Dilution Index and Second Concentration Moment $M$ as measured from the small scale direct numerical simulations (black solid line) and predictions with the SMM (red circles) for two different Péclet numbers $(P e=100$ and $P e=1000)$

of data are shown corresponding to measurements from the direct numerical solutions as well as predictions with our novel modified SMM, implementing the algorithm depicted in figure 5. Again, the match between measured values and those predicted by our effective model is good, matching well for both considered Péclet numbers over the full range of modeled times, which span pre-asymptotic to asymptotic. The fact that the SMM predictions match the DNS data well over all times for both Péclet numbers and both metrics, suggests that our proposed SMM is robust.

\subsection{Convergence test}

One of the critical features of the novel downscaling element of the proposed SMM is the number of points that are stored in matrix $\mathbf{P}\left(s_{i}\right)$ defined in equation (8), that is the number of points along a particle's trajectory that one stores 
between which to interpolate and reconstruct a particle's location. In this work we used $n=50$ and in the previous section demonstrated that this reconstructed the evolution of our chosen global mixing measures well.

To address the issue of how many points one should use in a more rigorous manner on Figure 8 we show the predicted evolution of the dilution index for varying $n$, all the way from $n=2$ (interpolating directly between a particle's inlet and outlet location) up to $n=50$. Results are shown for both Péclet numbers of interest with very similar trends. We focus only on the dilution index as trends for the second concentration moment are very similar. In both cases $n=2$ vastly underpredicts the evolution of the dilution index, meaning that the model predicts that the plume occupies less space than it should. Physically this makes sense as a direct interpolation from the inlet face to the outlet face does not allow the full pore volume to be occupied and unphysically restricts locations where particles can be positioned; that is particles cannot take an absolute $|y|$ value anywhere above $\bar{h}-h^{\prime}$, restricting particles to locations confined between the thinnest section of the pore and leaving a lot of empty space that cannot be filled. As $n$ increases more and more space can be filled and eventually appears to converge to reasonable values for $n=25$, the results of which are visually indistinguishable from $n=50$, which is in turn indistinguishable from the DNS results, as shown in Figure 7.

Likewise Fig 9 plots the mean absolute relative error $(M A R E)$, defined as,

$$
M A R E=\frac{1}{N} \sum_{i=1}^{N} \frac{\left|E\left(t_{i}\right)_{D N S}-E\left(t_{i}\right)_{S M M}\right|}{E\left(t_{i}\right)_{D N S}},
$$

where $E_{D N S}$ and $E_{S M M}$ are the dilution index measured from the fully resolved direct numerical simulations and predicted by the SMM respectively. Clearly the small values of $n$ do a terrible job, as was already visually evident from Figure 8 , with the results converging as $n$ increases. For our chosen value of $n=50$ the errors are less than $10^{-2}$ meaning that predicted and measured values of the dilution index are within $1 \%$ of another. Interestingly the MAREs for $P e=1000$ are lower than those for $P e=100$, which is the reverse of what one 

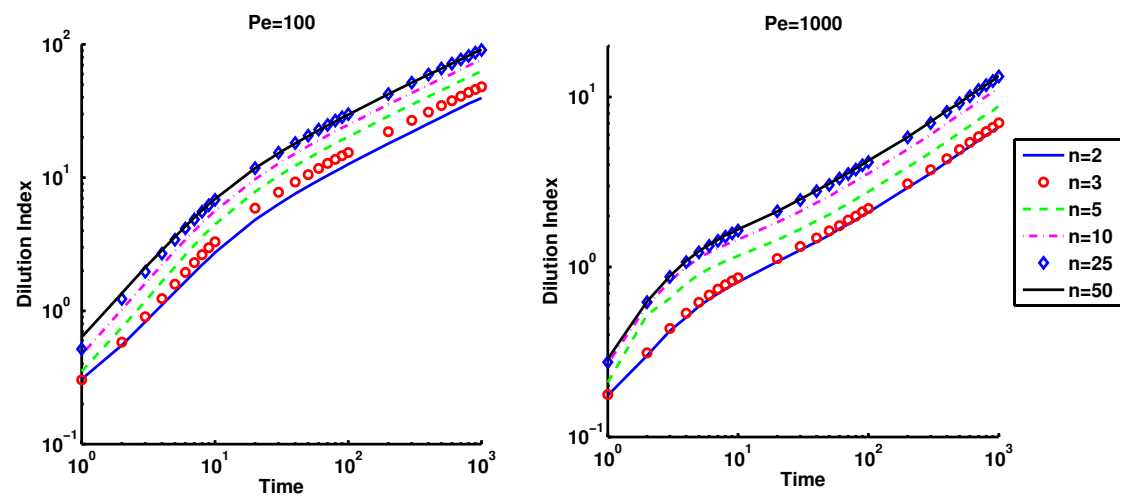

Figure 8: A comparison of the Dilution Index predicted with the SMM for varying values of $n$ the number of interpolation points used in the downscaling of the concentration field. Results are shown for both Péclet numbers $(P e=100$ and $P e=1000)$

might intuitively expect given that for $P e=1000$ correlation effects are known to be much more important in the context of SMMs. However, we believe that for $P e=100$ the location of a particle is less certain due to the nature of diffusion and so an accurate reconstruction of its path is more challenging. However, for both cases the $n=50$ case yields very satisfactory results.

\section{Conclusions}

We have introduced a novel variant of the Spatial Markov Model, a class of model that has previously been used with great success to predict effective transport across a wide range of applications of hydrologic interest. The novelty of the present SMM is that rather than focusing on only the travel time statistics of particles as they transition through space, we build the model by focusing on specific trajectories that particles can take, which includes information on both the current travel time a particle will have as well as the limited number of trajectories that it can sample during its next transition. This means that the model can be parameterized with information on transport across one unit cell, rather than two as has been the case for previous implementations. The SMM also brings with it a significant computational cost saving relative to running 


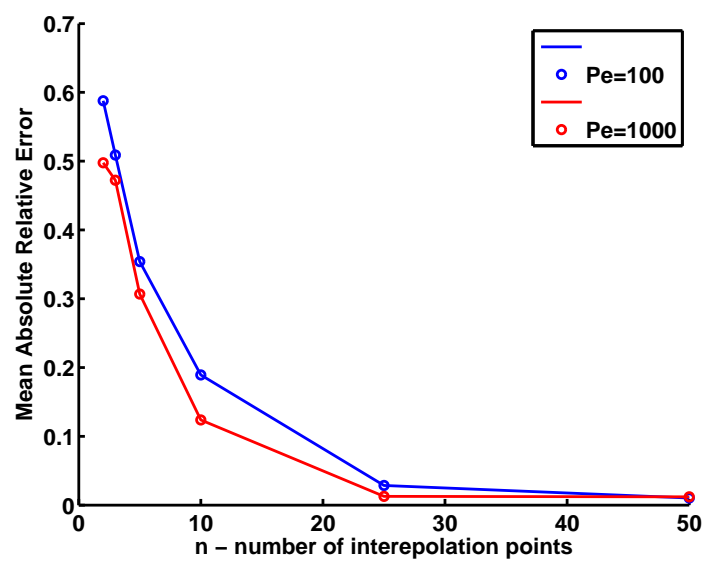

Figure 9: Mean Absolute Relative Error for the dilution index as a function of the number of interpolating points used for $P e=100$ and $P e=1000$

the full blown DNS. While the codes written for this paper were not written with optimization in mind, the SMM code is about 100 times faster than the DNS, offering a considerable speedup, which under further optimization could be improved even further.

In its current form, the model can be applied to a spatially periodic domain, recognizing that the potential trajectories a particle can sample are set by its position when it enters the domain. While the requirement for a periodic domain might be regarded as a potentially limiting feature of this model, it should also be noted that solving a closure problem on a periodic unit cell is required for many well established upscaling procedures. Several in depth discussions in the literature highlight that this should not be seen as a disadvantage of any of these approaches [e.g. 54, 65].

By applying the model to a simple benchmark periodic flow, we demonstrate that this novel SMM is able to faithfully represent effective transport for two Péclet numbers, $P e=100$ and $P e=1000$, chosen because these transition to the regime where previous studies have shown correlation effects to be important, requiring an upscaled model that accounts for these, which the SMM does.

Recognizing that we can store more details on the trajectories that particles 
take, including the specific location of a particle at fixed temporal increments along a trajectory, we also propose a methodology for using the modified SMM to downscale the mean effective model to provide a physically sound estimate of a particle's location at a specific time. By providing the approximate position of particles at a specific time we can reconstruct the full concentration field at a microscopic level and use this reconstructed concentration field to measure quantities that are sensitive to subscale fluctuations, such as the dilution index or moments of concentration. For our benchmark model, for both of the considered Péclet numbers we demonstrate that this methodology works well at predicting both the dilution index and the second moment of concentration. In all cases the comparison between the direct numerical simulations where all processes are fully resolved and the modified SMM are excellent. To our knowledge this is the first time that an SMM has been used to successfully predict the temporal evolution of global mixing metrics such as the dilution index and second moment of concentration, demonstrating its potential in this regard as well as opening an avenue for its potential use in predicting mixing driven reactive transport. Additionally the fact that our modified SMM appears to be able to predict volume averaged quantities associated with subscale fluctuations suggests that it may be a promising model that could be used in some hybrid manner to speed up the computation of closures that arise during formal upscaling, e.g., by volume averaging [e.g. 51]. We also envision that our approach can be extended to mixing within heterogeneous non periodic systems, possibly extending existing models recently developed for this purpose [e.g. 50]. Additionally, given structural similarities between our approach and streamline based upscaling approaches [e.g. 22], with some modification we may be able to readily integrate our methodologies into established codes and numerical frameworks. These are issues that must be addressed in future efforts to assess the full potential of our modified SMM framework.

Acknowledgments We would like to express thanks for financial support via NSF grants EAR-1351625, EAR-1417264 and EAR-1446236 


\section{References}

[1] I. Battiato, D. Tartakovsky, Applicability regimes for macroscopic models of reactive transport in porous media, Journal of Contaminant Hydrology 120 (2011) 18-26.

[2] I. Battiato, D. M. Tartakovsky, A. M. Tartakovsky, T. Scheibe, On breakdown of macroscopic models of mixing-controlled heterogeneous reactions in porous media, Advances in Water Resources 32 (11) (2009) 1664-1673.

[3] J. Bear, A. Cheng, Modeling Groundwater Flow and Contamimant Transport, Springer, 2010.

[4] D. A. Beard, Taylor dispersion of a solute in a microfluidic channel, Journal of Applied Physics 89 (8) (2001) 4667-4669.

[5] D. A. Benson, M. M. Meerschaert, Simulation of chemical reaction via particle tracking: Diffusion-limited versus thermodynamic rate-limited regimes, Water Resources Research 44 (12).

[6] B. Berkowitz, I. Dror, S. K. Hansen, H. Scher, Measurements and models of reactive transport in geological media, Reviews of Geophysics (2016) 2016RG000524.

[7] B. Bijeljic, A. Raeini, P. Mostaghimi, M. J. Blunt, Predictions of nonFickian solute transport in different classes of porous media using direct simulation on pore-scale images, Physical Review E 87 (1) (2013) 013011.

[8] D. Bolster, T. L. Borgne, M. Dentz, Solute dispersion in channels with periodically varying apertures, Physics of Fluids 21 (2009) 056601.

[9] D. Bolster, M. Dentz, J. Carrera, Effective two-phase flow in heterogeneous media under temporal pressure fluctuations, Water Resources Research 45 (5).

[10] D. Bolster, Y. Méheust, T. Le Borgne, J. Bouquain, P. Davy, Modeling preasymptotic transport in flows with significant inertial and trapping 
effects-the importance of velocity correlations and a spatial Markov model, Advances in Water Resources 70 (2014) 89-103.

[11] D. Bolster, F. Valdes-Parada, T. L. Borgne, M. Dentz, J. Carrera, Mixing in confined stratified aquifers, Journal of Contaminant Hydrology 120-121 (2011) 198-212.

[12] J. Bouquain, Y. Meheust, D. Bolster, P. Davy, The impact of inertial effects on solute dispersion in a channel with periodically varying aperture, Physics of Fluids 24 (2012) 083602.

[13] H. Brenner, Taylor dispersion in systems of sedimenting nonspherical brownian particles. I. Homogeneous, centrosymmetric, axisymmetric particles, Journal of Colloid and Interface Science 71 (2) (1979) 189-208.

[14] H. Brenner, Dispersion resulting from flow through spatially periodic porous media, Philosophical Transactions of the Royal Society of London. Series A 297 (1980) 81-133.

[15] J. Cao, P. Kitanidis, Pore-scale dilution of conservative solutes: An example, Water resources research 34 (8) (1998) 1941-1949.

[16] M. B. Cardenas, Three-dimensional vortices in single pores and their effects on transport, Geophysical Research Letters 35 (18).

[17] M. B. Cardenas, Direct simulation of pore level Fickian dispersion scale for transport through dense cubic packed spheres with vortices, Geochem., Geophs., Geosyst. 10 (2009) Q12014.

[18] K. Chaudhary, M. Cardenas, W. Deng, P. Bennett, Pore geometry effects on intrapore viscous to inertial flows and on effective hydraulic parameters, Water Resources Research 49 (2013) 1149-1162.

[19] K. Chaudhary, M. B. Cardenas, W. Deng, P. C. Bennett, The role of eddies inside pores in the transition from Darcy to Forchheimer flows, Geophysical Research Letters 38 (24). 
[20] S. Chilukuri, C. H. Collins, P. T. Underhill, Dispersion of flagellated swimming microorganisms in planar Poiseuille flow, Physics of Fluids (1994present) 27 (3) (2015) 031902.

[21] I. C. Christov, H. A. Stone, Shear dispersion in dense granular flows, Granular Matter 16 (4) (2014) 509-515.

[22] M. J. Crane, M. J. Blunt, Streamline-based simulation of solute transport, Water Resources Research 35 (10) (1999) 3061-3078.

URL http://dx.doi.org/10.1029/1999WR900145

[23] G. Dagan, Flow and transport in porous formations, Springer Science \& Business Media, 2012.

[24] P. de Anna, J. Jimenez-Martinez, H. Tabuteau, R. Turuban, T. Le Borgne, M. Derrien, Y. Meheust, Mixing and reaction kinetics in porous media: An experimental pore scale quantification, Environmental Science \& Technology 48 (1) (2013) 508-516.

[25] P. De Anna, T. Le Borgne, M. Dentz, A. M. Tartakovsky, D. Bolster, P. Davy, Flow intermittency, dispersion, and correlated continuous time random walks in porous media, Physical Review Letters 110 (18) (2013) 184502.

[26] M. Dentz, D. Bolster, Distribution-versus correlation-induced anomalous transport in quenched random velocity fields, Physical Review Letters 105 (24) (2010) 244301.

[27] M. Dentz, T. Le Borgne, A. Englert, B. Bijeljic, Mixing, spreading and reaction in heterogeneous media: A brief review, Journal of Contaminant Hydrology 120 (2011) 1-17.

[28] D. Ding, D. A. Benson, A. Paster, D. Bolster, Modeling bimolecular reactions and transport in porous media via particle tracking, Advances in Water Resources 53 (2013) 56-65. 
[29] B. Dykaar, P. Kitanidis, Macrotransport of a biologically reacting solute through porous media, Water Resources Research 32 (1996) 307-329.

[30] Y. Edery, H. Scher, B. Berkowitz, Particle tracking model of bimolecular reactive transport in porous media, Water Resources Research 46 (7).

[31] C. M. Gramling, C. F. Harvey, L. C. Meigs, Reactive transport in porous media: A comparison of model prediction with laboratory visualization, Environmental Science \& Technology 36 (11) (2002) 2508-2514.

[32] U. Hornung, Homogenization and Porous Media, Springer, 1997.

[33] M. P. Howard, A. Gautam, A. Z. Panagiotopoulos, A. Nikoubashman, Axial dispersion of Brownian colloids in microfluidic channels, Physical Review Fluids 1 (4) (2016) 044203.

[34] P. K. Kang, P. Anna, J. P. Nunes, B. Bijeljic, M. J. Blunt, R. Juanes, Pore-scale intermittent velocity structure underpinning anomalous transport through 3-D porous media, Geophysical Research Letters 41 (17) (2014) 6184-6190.

[35] P. K. Kang, M. Dentz, T. Le Borgne, R. Juanes, Spatial Markov model of anomalous transport through random lattice networks, Physical Review Letters 107 (18) (2011) 180602.

[36] P. K. Kang, T. Le Borgne, M. Dentz, O. Bour, R. Juanes, Impact of velocity correlation and distribution on transport in fractured media: Field evidence and theoretical model, Water Resources Research 51 (2) (2015) 940-959.

[37] P. Kitanidis, The concept of the dilution index, Water Resources Research 30 (1994) 2011-2026.

[38] P. Kitanidis, B. Dykaar, Stokes flow in a slowly varying two-dimensional periodic pore, Transport in Porous Media 26 (1997) 89-98.

[39] T. Le Borgne, M. Dentz, D. Bolster, J. Carrera, J.-R. De Dreuzy, P. Davy, Non-Fickian mixing: Temporal evolution of the scalar dissipation rate in 
heterogeneous porous media, Advances in Water Resources 33 (12) (2010) $1468-1475$.

[40] T. Le Borgne, M. Dentz, J. Carrera, Lagrangian statistical model for transport in highly heterogeneous velocity fields, Physical Review Letters 101 (2008) 090601.

[41] T. Le Borgne, M. Dentz, J. Carrera, Spatial Markov processes for modeling Lagrangian particle dynamics in heterogeneous porous media, Physical Review E 78 (2008) 026308.

[42] T. Le Borgne, M. Dentz, E. Villermaux, The lamellar description of mixing in porous media, Journal of Fluid Mechanics 770 (2015) 458-498.

[43] T. LeBorgne, D. Bolster, M. Dentz, P. de Anna, A. Tartakovsky, Effective pore-scale dispersion upscaling with a correlated CTRW approach, Water Resources Research 47 (2012) W12538.

[44] I. Lunati, S. Attinger, W. Kinzelbach, Macrodispersivity for transport in arbitrary nonuniform flow fields: Asymptotic and preasymptotic results, Water Resources Research 38 (10) (2002) 5-1.

[45] A. Paster, T. Aquino, D. Bolster, Incomplete mixing and reactions in laminar shear flow, Physical Review E 92 (1) (2015) 012922.

[46] A. Paster, D. Bolster, D. A. Benson, Connecting the dots: Semi-analytical and random walk numerical solutions of the diffusion-reaction equation with stochastic initial conditions, Journal of Computational Physics 263 (2014) 91-112.

[47] O. Plumb, S. Whitaker, Dispersion in heterogeneous porous media: 1. Local volume averaging and large-scale averaging, Water Resources Research 24 (1988) 913-926.

[48] J. Poate, T. Illangasekare, H. Kazemi, R. Kee, Pore Scale Phenomena: Frontiers in Energy and Environment, World Scientific, 2015. 
[49] S. Pope, Turbulent Flows, Cambridge University Press, 2000.

[50] G. Porta, B. Bijeljic, M. J. Blunt, A. Guadagnini, Continuum-scale characterization of solute transport based on pore-scale velocity distributions, Geophysical Research Letters 42 (18) (2015) 7537-7545.

[51] G. Porta, G. Ceriotti, J.-F. Thovert, Comparative assessment of continuum-scale models of bimolecular reactive transport in porous media under pre-asymptotic conditions, Journal of Contaminant Hydrology 185 (2016) 1-13.

[52] G. Porta, J.-F. Thovert, M. Riva, A. Guadagnini, P. Adler, Microscale simulation and numerical upscaling of a reactive flow in a plane channel, Physical Review E 86 (3) (2012) 036102.

[53] R. F. Probstein, Physicochemical Hydrodynamics: An Introduction, John Wiley \& Sons, 2005.

[54] P. Renard, G. De Marsily, Calculating equivalent permeability: a review, Advances in water resources 20 (5) (1997) 253-278.

[55] M. C. Richmond, W. A. Perkins, T. D. Scheibe, A. Lambert, B. D. Wood, Flow and axial dispersion in a sinusoidal-walled tube: Effects of inertial and unsteady flows, Advances in Water Resources 62 (2013) 215-226.

[56] H. Risken, Fokker-Planck Equation, Springer, 1984.

[57] J. Salles, J.-F. Thovert, R. Delannay, L. Prevors, J.-L. Auriault, P. Adler, Taylor dispersion in porous media. determination of the dispersion tensor, Physics of Fluids A 5 (10) (1993) 2348-2376.

[58] J. Sané, J. T. Padding, A. A. Louis, Taylor dispersion of colloidal particles in narrow channels, Molecular Physics 113 (17-18) (2015) 2538-2545.

[59] M. Siena, M. Riva, J. Hyman, C. Winter, A. Guadagnini, Relationship between pore size and velocity probability distributions in stochastically generated porous media, Physical Review E 89 (1) (2014) 013018. 
[60] N. Sund, D. Bolster, S. Mattis, C. Dawson, Pre-asymptotic transport upscaling in inertial and unsteady flows through porous media, Transport in Porous Media 109 (2) (2015) 411-432.

[61] N. L. Sund, D. Bolster, D. A. Benson, Testing the limits of the spatial Markov model for upscaling transport: The role of nonmonotonic effective velocity autocorrelations, Physical Review E 94 (4) (2016) 043107.

[62] N. L. Sund, D. Bolster, C. Dawson, Upscaling transport of a reacting solute through a periodically converging-diverging channel at pre-asymptotic times, Journal of Contaminant Hydrology 182 (2015) 1-15.

[63] G. Taylor, Dispersion of soluble matter in solvent flowing slowly through a tube, Proceedings of the Royal Society of London. Series A, 219 (1953) 186-203 186-203.

[64] B. D. Wood, F. Cherblanc, M. Quintard, S. Whitaker, Volume averaging for determining the effective dispersion tensor: Closure using periodic unit cells and comparison with ensemble averaging, Water Resources Research 39 (8).

[65] B. D. Wood, F. Cherblanc, M. Quintard, S. Whitaker, Volume averaging for determining the effective dispersion tensor: Closure using periodic unit cells and comparison with ensemble averaging, Water Resources Research 39 (8) (2003) n/a-n/a, 1210.

URL http://dx.doi.org/10.1029/2002WR001723 